\title{
Casein Kinases I and $2 \alpha$ Phosphorylate Oryza Sativa Pseudo-Response Regulator 37 (OsPRR37) in Photoperiodic Flowering in Rice
}

\author{
Choon-Tak Kwon', Bon-Hyuk Koo', Dami Kim', Soo-Cheul Yoo', and Nam-Chon Paek ${ }^{1, *}$
}

\begin{abstract}
Flowering time (or heading date) is controlled by intrinsic genetic programs in response to environmental cues, such as photoperiod and temperature. Rice, a facultative shortday (SD) plant, flowers early in SD and late in long-day (LD) conditions. Casein kinases (CKs) generally act as positive regulators in many signaling pathways in plants. In rice, Heading date 6 (Hd6) and Hd16 encode CK2 $\alpha$ and CKI, respectively, and mainly function to delay flowering time. Additionally, the major LD-dependent floral repressors Hd2/ Oryza sativa Pseudo-Response Regulator 37 (OsPRR37; hereafter PRR37) and Ghd7 also confer strong photoperiod sensitivity. In floral induction, Hd16 acts upstream of Ghd7 and CKI interacts with and phosphorylates Ghd7. In addition, Hd6 and Hd16 also act upstream of Hd2. However, whether $C K I$ and $C K 2 \alpha$ directly regulate the function of PRR37 remains unclear. Here, we use in vitro pull-down and in vivo bimolecular fluorescence complementation assays to show that $C K I$ and $C K 2 \alpha$ interact with PRR37. We further use in vitro kinase assays to show that $C K I$ and $C K 2 \alpha$ phosphorylate different regions of PRR37. Our results indicate that direct posttranslational modification of PRR37 mediates the genetic interactions between these two protein kinases and PRR37. The significance of CK-mediated phosphorylation for PRR37 and Ghd7 function is discussed.
\end{abstract}

\section{INTRODUCTION}

In plants, complex interactions between endogenous circadian clock components and environmental factors trigger flowering; these environmental factors include seasonal changes in day length (photoperiod) and temperature. To date, research in

1Department of Plant Science, Plant Genomics and Breeding Institute, and Research Institute for Agriculture and Life Sciences, Seoul National University, Seoul 151-921, Korea, '2Department of Bioresource and Rural System of Engineering, Hankyong National University, Ansung 456-749, Korea

*Correspondence: ncpaek@snu.ac.kr

Received 17 September, 2014; revised 17 October, 2014; accepted 17 October, 2014; published online 26 November, 2014

Keywords: casein kinase I, casein kinase $2 \alpha$, flowering time, OsPRR37, phosphorylation, rice model systems and crops has identified many regulatory genes controlling flowering time (also known as heading date), for example in the dicot Arabidopsis thaliana and the monocot rice (Oryza sativa).

Cultivated rice is a facultative short-day (SD) plant that flowers early in SD (< 10-h light/day) and late in long day (LD; > 14h light/day) conditions (Izawa, 2007; Tsuji et al., 2008). Modern rice cultivation spans geographical latitudes from $53^{\circ} \mathrm{N}$ to $40^{\circ} \mathrm{S}$ and photoperiod sensitivity affects adaptation for growth at these different latitudes, significantly affecting grain yield (Izawa, 2007). For example, japonica rice in the northern-limit regions $\left(>40^{\circ} \mathrm{N}\right)$ generally flowers extremely early, which results in proper grain production under natural LD conditions in the short summer period (Fujino and Sekiguchi, 2005; Wei et al., 2008). In Arabidopsis, four major pathways regulate flowering time: photoperiod, gibberellin, vernalization, and autonomous pathways. In LD conditions, the GIGANTEA (GI)-CONSTANS (CO)-FLOWERING LOCUS T (FT) module is the major photoperiod pathway for floral induction (Huq et al., 2000; Kardailsky et al., 1999; Kobayashi et al., 1999; Park et al., 1999). The florigen FT moves from the leaves to the shoot apex and binds to the bZIP transcription factor FD to activate the expression of floral meristem-identity genes (Abe et al., 2005; Corbesier et al., 2007). In rice, OsGl-Heading date 1 (Hd1)-Heading date $3 a$ $(\mathrm{Hd} 3 \mathrm{a})$ form a conserved pathway that functions in floral induction, mainly in SD (Hayama et al., 2003; Yano et al., 2000). In addition, the OSMADS50-Early heading date 1 (Ehd1)-Rice flowering locus T1 (RFT1) pathway also functions in floral induction, mainly in LD (Doi et al., 2004; Komiya et al., 2009). The rice florigens Hd3a and RFT1 activate the expression of the floral meristem-identity genes OsMADS14 and OsMADS15 (Komiya et al., 2008). In the two principal pathways of floral induction, Days to heading 8 (DTH8)/Grain number, plant height and heading date 8 (Ghd8)/Hd5 suppresses Ehd1 expression to inhibit flowering in LD (Fujino et al., 2013; Wei et al., 2010; Yan et al., 2011). Ghd7 functions as one of the major LDdependent floral repressors, downregulating Ehd1 expression (Xue et al., 2008). By contrast, Rice Indeterminate 1 (OsID1)/ Ehd2/RID1 (Matsubara et al., 2008b; Park et al., 2008; Wu et al., 2008) and Ehd3 (Matsubara et al., 2011) function as major positive regulators, upregulating Ehd1 expression to promote flowering in LD. Recent work has identified several genes upstream of $H d 1$ and Ehd1; for example, Hd17/OsELF3, the rice ortholog of Arabidopsis ELF3, functions as a floral inducer by downregulating Ghd7 expression (Matsubara et al., 2012; Saito 
et al., 2012; Yang et al., 2013; Zhao et al., 2012). Ehd4 acts upstream of Ehd1 and positively regulates the expression of $\mathrm{Hd} 3 \mathrm{a}$ and RFT1 by increasing Ehd1 expression (Gao et al., 2013). By contrast, DTH2, which encodes a CO-like protein, promotes flowering by upregulating the expression of $\mathrm{Hd} 3 \mathrm{a}$ and RFT1 independent of Hd1 and Ehd1 (Wu et al., 2013).

In Arabidopsis, direct protein-protein interactions play crucial roles in the control of flowering time. For example, initiation of floral development in the shoot apex requires the interaction between FT and FD (Abe et al., 2005). In LD, FLAVINBINDING, KELCH REPEAT, F-BOX 1 (FKF1) interacts with GI and the FKF1-GI complex activates $C O$ expression in the late afternoon (Sawa et al., 2007). FKF1 also interacts with CO through its LOV domain and stabilizes $\mathrm{CO}$ binding to the $F T$ promoter (Song et al., 2012). During the night, CONSTITUTIVE PHOTOMORPHOGENIC1 (COP1) interacts with and destabilizes CO. In SD, ELF3 interacts with COP1 and Gl; this allows COP1 to ubiquitinate ELF3 and GI for COP1-ELF3-GI degradation via the 26S proteasome (Yu et al., 2008). Furthermore, ELF3 also forms a complex with LUX ARRHYTHMO (LUX) and ELF4; the ELF4-ELF3-LUX complex forms transiently at dusk to downregulate the expression of PHYTOCHROMEINTERACTING FACTOR 4 (PIF4) and PIF5 for diurnal hypocotyl growth (Herrero et al., 2012; Nusinow et al., 2011). In contrast to Arabidopsis, most of the protein-protein interactions between flowering-time regulators in rice remain to be examined. One recent example showed that $\mathrm{Hd} 3 \mathrm{a}$ binds to 14-3-3; their complex moves to the nucleus and interacts with OsFD1 to induce floral meristem-identity genes (Taoka et al., 2011).

The highly conserved serine/threonine-specific casein kinases (CKs) control various signal transduction processes in eukaryotes (Knippschild et al., 2005; Mulekar and Huq, 2014). In plants, CKI and CK2 affect the regulation of flowering time and circadian rhythm. In Arabidopsis, CK1.3 and CK1.4 have important roles in the regulation of bluelight signaling and circadian rhythm by decreasing the stability of cryptochrome 2 through phosphorylation (Tan et al., 2013). Moreover, the highly conserved circadian clock component CK2 positively regulates the stability of the clock oscillators CIRCADIAN CLOCK ASSOCIATED 1 (CCA1) (Daniel et al., 2004; Lu et al., 2011; Portoles and Mas 2010; Sugano et al., 1998) and rice LATE ELONGATED HYPOCOTYL (OsLHY) (Ogiso et al., 2010). Rice Hd6, which encodes the a subunit of CK2 (CK2 $\alpha)$, does not control circadian rhythm but delays flowering time in LD conditions in the presence of functional $\mathrm{Hd} 1$, indicating that Hd6 genetically acts upstream of $\mathrm{Hd} 1$ in LD-dependent floral repression (Ogiso et al., 2010). However, CK2 $\alpha$ does not interact with or phosphorylate Hd1 (Ogiso et al., 2010). Also, Hd16/Early flowering1 (EL1), which encodes CKI, not only represses gibberellin signaling by phosphorylating SLENDER RICE1 (SLR1) but also inhibits LD-dependent flowering by phosphorylating Ghd7, resulting in the suppression of Ehd1, Hd3a, and RFT1 (Dai and Xue, 2010; Hori et al., 2013; Kwon et al., 2014).

Pseudo-Response Regulators (PRRs) are essential circadian clock components in Arabidopsis (Alabadi et al., 2001; Farre and Kay, 2007; Ito et al., 2009; Kaczorowski and Quail, 2003; Nakamichi et al., 2007; Yamamoto et al., 2003). The five PRR genes (PRR1/TIMING OF CAB EXPRESSION 1 [TOC1], PRR3, PRR5, PRR7, and PRR9) show differential circadian expression from dawn to dusk (Matsushika et al., 2000). In planta, all of the PRRs are phosphorylated, which may be essential for their function. However, the protein ki- nases responsible for the phosphorylation of PRRs have not yet been identified (Fujiwara et al., 2008). Rice also has five orthologs of Arabidopsis PRR genes: OsPRR1/OsTOC1, OsPRR37, OsPRR59, OsPRR73, and OsPRR95 (Murakami et al., 2005; 2007). The rice ortholog of Arabidopsis PRR7, Hd2/OsPRR37 (hereafter PRR37) functions as one of the major LD-dependent floral repressors, together with Ghd7 (Xue et al., 2008), both of which play an important role in photoperiod sensitivity in rice (Koo et al., 2013; Murakami et al., 2005; Yan et al., 2013).

Here, we show the direct interactions of $\mathrm{Hd} 6 / \mathrm{CK} 2 \alpha$ and Hd16/CKI with Hd2/PRR37 in vitro and in vivo. We also show that these two CKs phosphorylate different regions of PRR37. Our study provides new insights into the role of CK-mediated phosphorylation of PRRs in LD-dependent floral repression in rice and in other plants.

\section{MATERIALS AND METHODS}

Plasmid construction and recombinant protein production The cDNAs of Hd6, PRR37 and partial region of OsLHY were obtained by RT-PCR with total RNA extracted from the leaves of the japonica-indica hybrid rice cultivar 'Milyang23' using the genespecific primers (Supplementary Table S1). The Hd6 and PRR37 were ligated into the pCR8/GW/TOPO plasmid (Invitrogen, USA). Then, the Hd6 and PRR37 DNA fragments in plasmids were digested with EcoRl. For expression in $E$. coli, the fragments were inserted into pGEX-4T-1 for glutathione S-transferase (GST)-tagged Hd6/CK2 $\alpha$ protein (GST-CK2 $\alpha$ ) and the modified pET28a for His(6x)-Maltose binding protein (HisMBP)- tagged PRR37 protein (HisMBP-PRR37) and HisMBP-LHYc [C-terminal region (520-719 residues) of OsLHY; Ogiso et al., 2010]. Proteins were expressed in Rosetta 2 cells after induction by $0.5 \mathrm{mM}$ isopropyl $\beta$-D-1-thiogalactopyranoside (IPTG) in SOB liquid media for $16 \mathrm{~h}$ at $20^{\circ} \mathrm{C}$. After spin-down to remove the SOB media, cells were broken by sonication, and the tagged proteins were purified with GST-Bind agarose resin (Elpis Biotech, Korea) or His-Bind agarose resin (Elpis Biotech, Korea) according to the manufacturers' instructions. The GST-tag was removed from eluted GSTCK2 $\alpha$ fusion protein by biotinylated thrombin protease (Merck, Germany) for in vitro kinase assay. To remove the thrombin, GST-CK2 $\alpha$ eluate was incubated with streptavidin agarose (Merck, Germany) for 30 min at room temperature. The eluates were concentrated using Amicon Ultra (Millipore, Germany). The cDNA of EL1 was acquired from total RNA in leaf blades of rice cultivar 'Nipponbare'. Recombinant His(6x)-tagged EL1/ CKI protein (His-CKI) was expressed as described earlier (Kwon et al., 2014).

In vitro pull-down assay

Eluted HisMBP proteins (a negative control) and HisMBPPRR37 were incubated with GST-CK2 $\alpha$ or His-CKI in GSTBind Agarose Resin (Elpis Biotech, Korea) or MBP-Bind Agarose Resin (Elpis Biotech, Korea), respectively, at $4^{\circ} \mathrm{C}$ for 1 h. The resin was washed four times in GST pull-down washing buffer $(50 \mathrm{mM}$ Tris- $\mathrm{HCl}, \mathrm{pH} 7.5,200 \mathrm{mM} \mathrm{NaCl}, 0.5 \mathrm{mM} \beta-$ mercaptoethanol, $1 \%$ Triton $\mathrm{X}-100$ and $0.2 \%$ glycerol) or MBP pull-down washing buffer $(20 \mathrm{mM}$ Tris- $\mathrm{HCl}, \mathrm{pH} 7.4,200 \mathrm{mM}$ $\mathrm{NaCl}, 10 \mathrm{mM} \beta$-mercaptoethanol, $1 \mathrm{mM}$ EDTA). To boil the proteins bound to the resin, $5 x$ SDS-PAGE loading buffer was added and heated at $100^{\circ} \mathrm{C}$ for $4 \mathrm{~min}$. Proteins were resolved by SDS-PAGE and immunoblotted using antibodies against GST (Santa Cruz Biotechnology, USA), MBP (Santa Cruz Biotechnology, USA), and His(6x)-tag (Abcam, USA) 
Subcellular localization and bimolecular fluorescence complementation (BiFC) assays

For YFP-tagged and partial YFP-tagged PRR37 constructs, PCR-amplified PRR37 cDNA was ligated into pCR8/GW/TOPO plasmid (Invitrogen, USA). Each cDNA of PRR37, EL1 or Hd6 was cloned into the vector with CaMV $35 S$ promoter: YFP (pEarlyGate101 or pEarlyGate104) and the BiFC Gateway vectors to examine their subcellular localization and in vivo interaction (Citovsky et al., 2006). PRR37 clones were fused into four BiFC plasmid sets: pSAT5-DEST-cEYFP(175-end)C1(B) (pE3130), pSAT5(A)-DEST-cEYFP(175-end)-N1 (pE3132), pSAT4(A)-DEST-nEYFP(1-174)-N1 (pE3134), and pSAT4DEST-nEYFP(1-174)-C1 (pE3136), to generate cYFP-PRR37, PRR37-cYFP, PRR37-nYFP, and nYFP-PRR37, respectively. The same method was used for partial YFP-tagged EL1/CKI and $\mathrm{Hd} 6 / \mathrm{CK} 2 \alpha$ constructs. Each pair of recombinant plasmids encoding $n E Y F P$ and cEYFP fusions was mixed $1: 1(\mathrm{w} / \mathrm{w})$, cobombarded into onion epidermal layers using a DNA particle delivery system (Biolistic PDS-1000/He, BioRad, USA), and incubated on 0.5x Murashige and Skoog (MS) solid media in the presence or absence of MG132 $(50 \mu \mathrm{M})$ for $16-24 \mathrm{~h}$ at $22^{\circ} \mathrm{C}$ under light or dark incubation, followed by observation and image analysis using a Confocal Laser Scanning Microscope II (LSM710, Carl Zeiss, Germany).

\section{In vitro kinase assay}

To do the in vitro kinase assay, a $50 \mu$ reaction containing approximately $1.0 \mu \mathrm{g} / \mu \mathrm{l}$ of the recombinant kinase (rCK2 $\alpha$ or His$\mathrm{CKI}$ ) and $1.5 \mu \mathrm{g} / \mu \mathrm{l}$ of the substrate in $50 \mathrm{mM}$ Tris- $\mathrm{HCl}, \mathrm{pH} 7.5$, $0.1 \mathrm{mM}$ EGTA, $10 \mathrm{mM}$ MgAc, 2 mM HEPES, $0.1 \mathrm{mM}$ ATP, 2.5 $\mathrm{mM} \mathrm{MgCl} 2$ and $10 \mu \mathrm{Ci}\left[\gamma_{-}{ }^{32} \mathrm{P}\right] A T P$ (Izotop, Hungary) was incubated at $30^{\circ} \mathrm{C}$ for $30 \mathrm{~min}$. To stop the reaction, 5x SDS-PAGE loading buffer was added. After the separation of proteins by SDS-PAGE, the ${ }^{32} \mathrm{P}$-labeled proteins were visualized by autoradiography. This assay was modified from previous research (Kang et al., 2013; Youn et al., 2013)

\section{RESULTS}

\section{CK2 $\alpha$ and $C K I$ interact with PRR37 in vitro}

The suppression of flowering by $\mathrm{Hd} 6$ and $\mathrm{Hd} 16$ in non-inductive LD conditions requires functional Hd2 (Hori et al., 2013; Shibaya et al., 2011; Yamamoto et al., 2000), indicating that the floral repressor $\mathrm{Hd} 2$ acts downstream of $\mathrm{Hd} 6$ and $\mathrm{Hd} 16$ in the same pathway regulating rice flowering. $\mathrm{Hd} 2, \mathrm{Hd} 6$, and $\mathrm{Hd} 16$ encode PRR37, CK2 $\alpha$, and CKI, respectively, (Hori et al., 2013; Koo et al., 2013; Takahashi et al., 2001). Also, in Arabidopsis, PRR7 is phosphorylated by an as-yet unknown protein kinase(s) (Fujiwara et al., 2008). Thus, we hypothesized that the genetic interactions of $\mathrm{Hd} 2, \mathrm{Hd} 6$, and $\mathrm{Hd} 16$ may involve the phosphorylation of PRR37 by CK2 $\alpha$ or CKI, via direct interaction. To examine this hypothesis, we first performed in vitro pulldown assays to examine the interactions of CK2 $\alpha$-PRR37 and CKI-PRR37. To this end, we purified four recombinant fusion proteins, the N-terminal GST-tagged CK2 $\alpha$ (GST-CK2 $\alpha$ ), GST (a negative control), His(6x)-MBP-tagged PRR37 (HisMBPPRR37), and HisMBP (a negative control) (see "Materials and Methods"). When GST-CK2 $\alpha$ was pulled down with anti-GST antibody-conjugated agarose resins, HisMBP-PRR37 coimmunoprecipitated, but not the negative control HisMBP (Fig. $1 \mathrm{~A})$, indicating that PRR37 directly interacts with $\mathrm{CK} 2 \alpha$ in vitro. Furthermore, when HisMBP-PRR37 was pulled down with antiMBP antibody-conjugated agarose resins, His-CKI also coimmunoprecipitated with HisMBP-PRR37, but not with HisMBP
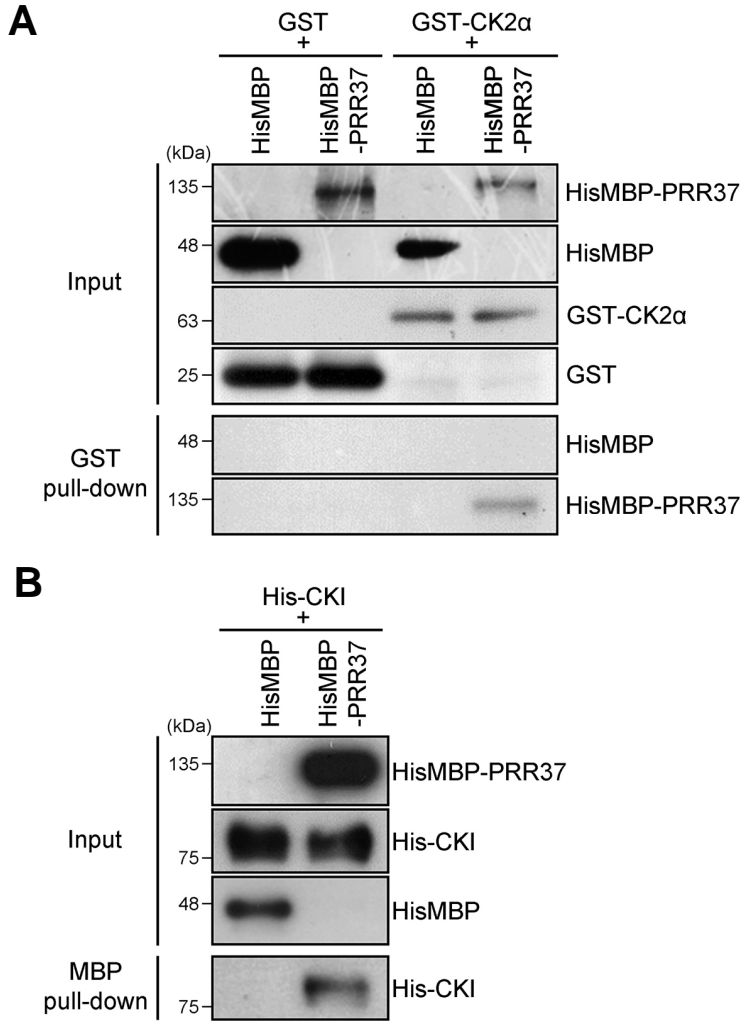

Fig. 1. PRR37 physically interacts with $\mathrm{CK} 2 \alpha$ and $\mathrm{CKI}$ in vitro. (A) Interaction between GST-CK2 $\alpha$ and HisMBP-PRR37 by in vitro pull-down assay using GST-Bind agarose resin. GST and GST-CK2 $\alpha$ were detected using anti-GST antibody. HisMBP and HisMBPPRR37 (input) were detected by anti-His antibody. After GST pulldown, HisMBP and HisMBP-PRR37 were detected by anti-MBP antibody. (B) His-CKI and HisMBP-PRR37 co-immunoprecipitated using MBP-Bind agarose resin. After MBP pull-down, His-CKI was detected by anti-His antibody. These experiments were repeated at least twice with the same results.

(Fig. 1B). These results show that $\mathrm{Hd} 6 / \mathrm{CK} 2 \alpha$ and $\mathrm{Hd} 16 / \mathrm{CKI}$ likely interact with Hd2/PRR37 for floral repression.

CK2 $\alpha$ and $C K I$ interact with PRR37 in vivo

To examine their potential interactions in vivo, we first examined the subcellular localization of CK2 $\alpha$ and PRR37, as previous work showed that CKI localizes to the nucleus (Dai and Xue, 2010). To examine the subcellular localization of CK2 $\alpha$ and PRR37, we constructed C-terminal yellow fluorescent protein (YFP)-tagged CK2 $\alpha$ (CK2 $\alpha-Y F P)$ and $N$-terminal YFPtagged PRR37 (YFP-PRR37) and bombarded these plasmids into onion epidermal cell layers. In these cells, we observed strong YFP signals in the nuclei expressing the CK2 $\alpha$-YFP and YFP-PRR37 proteins (Fig. 2A), indicating that CKI, CK2 $\alpha$, and PRR37 all localize to the nucleus.

To confirm the interactions of CK2 $\alpha$-PRR37 and CKI-PRR37 in vivo, we performed bimolecular fluorescence complementation (BiFC) assays. In the negative control experiment, we detected no YFP fluorescence in cells co-expressing nYFP and cYFP-PRR37 plasmids (Fig. 2B). In the positive control experiment, we observed YFP signals as speckles in the nucleus in cells co-expressing the known interactors nYFP-ELF3 and 
$\boldsymbol{A}$

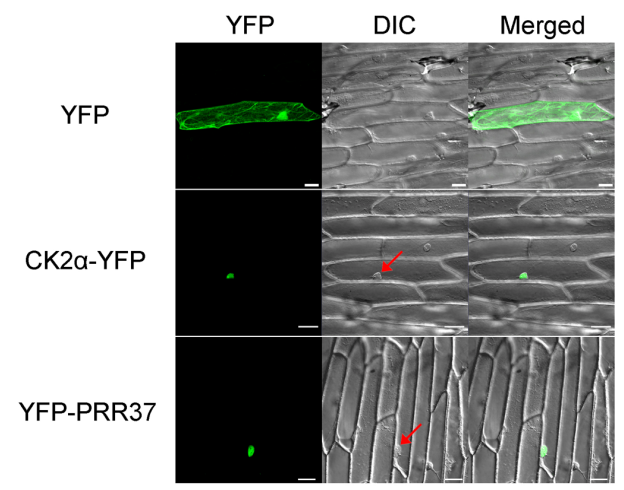

B

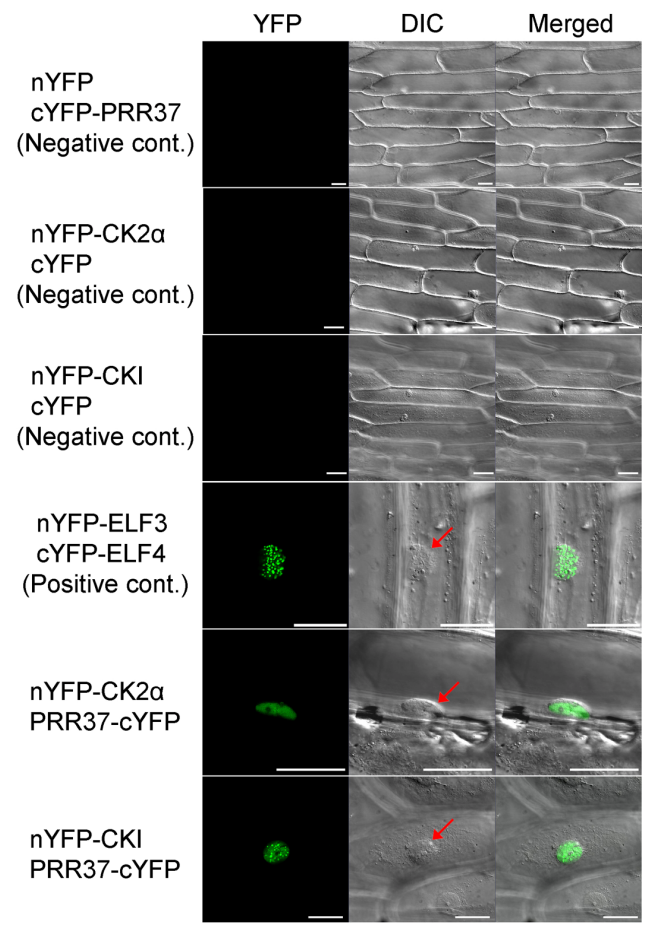

Fig. 2. PRR37 interacts with both $\mathrm{CK} 2 \alpha$ and $\mathrm{CKI}$ in vivo. (A) Subcellular localization of CK2 $\alpha$-YFP and YFP-PRR37 in onion epidermal cells. The panels show YFP, differential interference contrast (DIC) and merged images of onion epidermal cells. Empty vector (pEarlygate101) was used as the YFP control. Fluorescent signals of CK2 $\alpha$-YFP and YFP-PRR37 appeared in the nucleus. (B) Empty BiFC plasmids (nYFP and cYFP-PRR37; nYFP- CK2 $\alpha$ and cYFP; nYFP-CKI and cYFP) were used as a negative control. The YFP signals of co-expressed nYFP-AtELF3 and cYFP-AtELF4 (a positive control; At, Arabidopsis thaliana) were detected in the nucleus, as described (Herrero et al., 2012). For CK2 $\alpha$-PRR37 and CKI-PRR37 interactions, nYFP-CK2 $\alpha$ and nYFP-CKI were co-bombarded with PRR37-cYFP. BiFC analysis by transiently expressing nYFP-CK2 $\alpha$ and nYFP-CKI with PRR37-cYFP indicated the interaction of nYFPCK2 $\alpha$ and nYFP-CKI with PRR37-cYFP in nucleus. Red arrows indicate the nucleus. These experiments were repeated at least three times with the same results. Scale bar $=50 \mu \mathrm{m}$.

cYFP-ELF4 (Herrero et al., 2012; Nusinow et al., 2011) (Fig. $2 \mathrm{~B})$. Finally, we found that the onion epidermal cells coexpressing nYFP-CK2 $\alpha$ and PRR37-cYFP showed YFP fluo- rescence in the nucleus, as did cells co-expressing nYFP-CKI and PRR37-cYFP (Fig. 2B), indicating that CK2 $\alpha$ and CKI directly interact with PRR37 in the nucleus.

\section{CK2 $\alpha$ and CKI phosphorylate PRR37}

CK2 $\alpha$ and $\mathrm{CKI}$ can phosphorylate specific substrates; CK2 $\alpha$ phosphorylates OsLHY in the circadian clock and flowering regulation (Ogiso et al., 2010), and CKI phosphorylates SLR1 in GA signaling and Ghd7 in flowering regulation (Dai and Xue, 2010; Hori et al., 2013). To examine whether these two protein kinases can phosphorylate PRR37, we conducted in vitro kinase assays with HisMBP-PRR37, GST-CK2 $\alpha$, and His-CKI, using $\left[\gamma^{32}\right.$ P $]$ ATP as the label. For the GST-CK2 $\alpha$ fusion protein, we used a biotinylated thrombin protease to remove the GSTtag before conducting the kinase assay. For in vitro kinase assays, we confirmed that recombinant $\mathrm{CK} 2 \alpha$ (rCK2 $\alpha$ ) phosphorylated the positive control HisMBP-LHYc, the C-terminal region (520-719 residues) of OsLHY (Ogiso et al., 2010) (Fig. $3 A)$. The kinase assays revealed that $\mathrm{rCK} \alpha$ (Fig. $3 \mathrm{~A})$ and HisCKI (Fig. 3A), can phosphorylate HisMBP-PRR37, but do not phosphorylate the negative control HisMBP. His-CKI also autophosphorylated, as previously reported (Kwon et al., 2014) (Fig. 3B).

To determine the phosphorylation sites in PRR37, we first examined the PRR37 sequence with a casein kinase-specific phosphorylation site prediction algorithm, KinasePhos 2.0 (http://kinasephos2.mbc.nctu.edu.tw/). KinasePhos predicted 46 phosphorylated amino acids (aa) in PRR37, 36 serines and 10 threonines (Fig. 4A and Supplementary Fig. S1). To investigate the region of PRR37 phosphorylated by CK2 $\alpha$ and/or CKI, we divided PRR37 (742 total residues) into three segments, the N-terminal (PRR37n; aa 1-200, including the Pseudo Receiver [PR] domain), middle (PRR37m; aa 201-480), and the Cterminal segments (PRR37c; aa 481-742, including the CONSTANS, CO-like, and TOC1 [CCT] domain) (Fig. 4A). We fused these three segments of PRR37 to the N-terminal HisMBP-tag and purified the fusion proteins from $E$. coli. In vitro kinase assays revealed that CK2 $\alpha$ and CKI both phosphorylated PRR37m (Fig. 4B). However, only CKI phosphorylated PRR37c, which includes the CCT domain. Also, neither CK2 $\alpha$ nor CKI phosphorylated PRR37n, which includes the PR domain (Fig. 4B). These data suggest that CK2 $\alpha$ and CKI phosphorylate different residues of PRR37, which likely affects the activity and stability of PRR37, similar to Arabidopsis PRR proteins (Fujiwara et al., 2008).

\section{DISCUSSION}

Arabidopsis PRR7, an ortholog of rice PRR37, functions as an important component of the circadian clock (Nakamichi et al. 2005; 2007; Salome and McClung, 2005). In monocot plants, such as rice, wheat, barley, and sorghum, natural mutations of PRR37 orthologs affect seasonal or regional adaptation by modulating photoperiod sensitivity and flowering time, to maximize plant survival and grain yield (Beales et al., 2007; Koo et al., 2013; Murphy et al., 2011; Turner et al., 2005). The Arabidopsis prr7 loss-of-function mutants flower slightly late in inductive LD conditions, but the rice prr37 knockout mutants flower early in non-inductive LD conditions (Koo et al., 2013; Nakamichi et al., 2007). These results suggest that the regulatory roles of the PRR37 orthologs in growth and development have diverged in monocot and dicot plants.

In Arabidopsis, posttranslational modification of circadian clock components, such as CCA1 and PRR proteins (PRR1/TOC1, 
$\boldsymbol{A}$

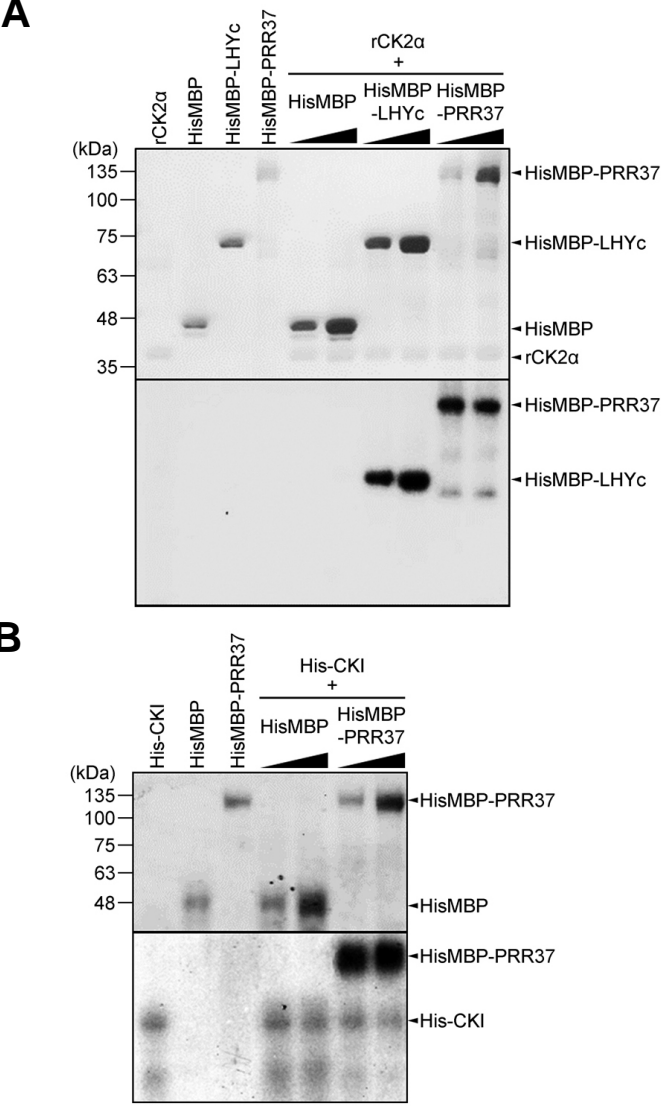

Fig. 3. $\mathrm{CK} 2 \alpha$ and $\mathrm{CKI}$ phosphorylates PRR37. (A) In vitro kinase assay using recombinant $\mathrm{CK} 2 \alpha(\mathrm{rCK} 2 \alpha)$ showed phosphorylation of HisMBP-LHYc [a positive control; C-terminal region (aa 520719) of OsLHY; Ogiso et al., 2010] and HisMBP-PRR37 proteins. HisMBP was used as a negative control. (B) In vitro kinase assay showed phosphorylation of HisMBP-PRR37 by His-CKI and autophosphorylation of His-CKI. Input of each protein was shown by Coomassie blue staining (upper panels), and phosphorylated proteins were detected by $\left[\gamma^{32} \mathrm{P}\right] \mathrm{ATP}$ autoradiography (lower panels). The black triangles above the panels indicate concentrations of substrates. These results were reproduced at least two times with the same results.

PRR3, PRR5, PRR7, and PRR9), influences protein activity and stability, via phosphorylation and selective proteolysis (Fujiwara et al., 2008). Phosphorylated TOC1 and PRR5 strongly interact with the F-box protein ZEITLUPE (ZTL), indicating that their regulation by proteolysis modulates circadian rhythm (Fujiwara et al., 2008). Moreover, phosphorylated PRR5 promotes the phosphorylation and nuclear accumulation of TOC1 (Wang et al., 2010). Nevertheless, the protein kinase(s) responsible for phosphorylating PRR proteins have not yet been identified. In this study, we show that in rice, the protein kinases CKI and CK2 $\alpha$ directly modify PRR37 at the posttranslational level (Figs. 1,2 , and 3 ).

In floral repression under non-inductive LD conditions, Hd6/CK2 $\alpha$ may phosphorylate downstream LD-dependent floral repressor(s). Several studies have reported the epistatic interactions between $H d 2 / P R R 37$ and other flowering-time regulators in rice. First, genetic studies showed that $\mathrm{Hd} 6$ acts upstream of $\mathrm{Hd} 2$ to delay flowering time, because $h d 2$ is
$\boldsymbol{A}$
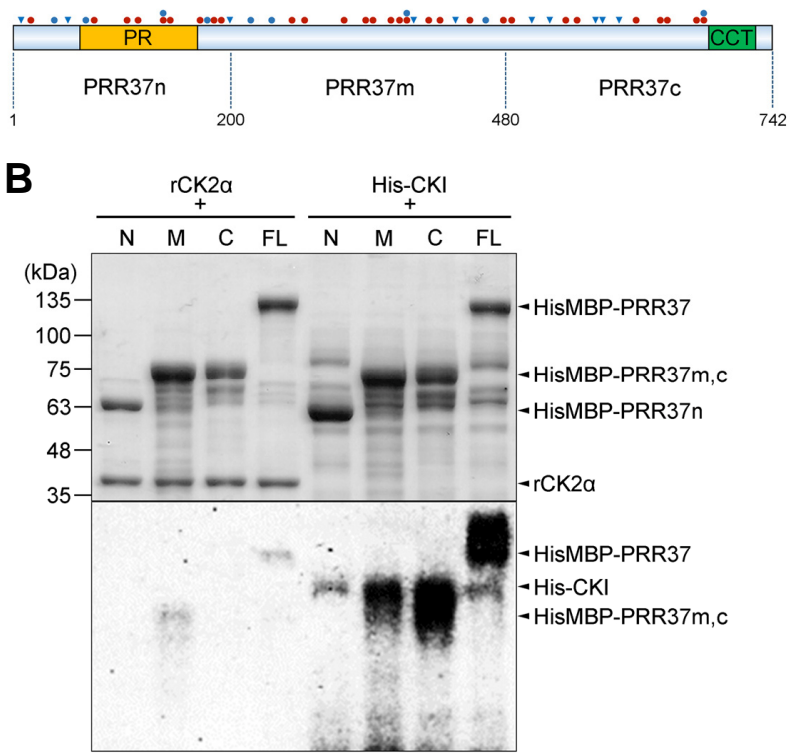

Fig. 4. CK2 $\alpha$ and $\mathrm{CKI}$ phosphorylate specific regions of PRR37. (A) The protein structure and predicted phosphorylation sites of PRR37 from rice cultivar 'Milyang23'. PR in yellow indicates a pseudo receiver (PR) domain and CCT in green indicates a CONSTANS, CO-like, and TOC1 (CCT) domain. The three partial PRR37 proteins were: PRR37n (1-200 aa), PRR37m (201-480 aa) and PRR37c (481-742 aa). Circles represent possible phosphorylation sites at serine by CKI (red) and CK2 (blue) residues. Inverted triangles indicate possible phosphorylation sites at threonine by $\mathrm{CKI}$ (red) and CK2 (blue). The phosphorylation prediction was obtained from KinasePhos 2.0 (http://kinasephos2.mbc.nctu.edu.tw/). (B) In vitro kinase assay of PRR37n (N), PRR37m (M), PRR37c (C) and full-length PRR37 (FL) proteins by rCK2 $\alpha$ and His-CKI. PRR37m and PRR37 were phosphorylated by rCK2 $\alpha$. Input of each protein was shown by Coomassie blue staining (upper panels), and phosphorylated proteins were detected by $\left[\gamma_{-}{ }^{32} \mathrm{P}\right]$ ATP autoradiography (lower panels). PRR37m, PRR37c and PRR37 were phosphorylated by His-CKI. Self-phosphorylation of His-CKI was also detected. These experiments were repeated at least three times with the same results.

epistatic to Hd6 or hd6 in LD conditions (Yammamoto et al., 2000). Thus, we postulate that CK2 $\alpha$ regulates PRR37 at the posttranslational level, because CK2 $\alpha$ interacts with and phosphorylates PRR37 (Figs. 1A, 2A, and 3A). Ogiso et al. (2010) reported that the LD-dependent floral repression of $\mathrm{Hd} 6$ requires functional $\mathrm{Hd} 1$ because hd1 is epistatic to Hd6 or hd6 in LD conditions, but CK2 $\alpha$ does not interact with or phosphorylate $\mathrm{Hd} 1$. Thus, they speculated that $\mathrm{Hd} 1$ activity may be regulated by an unknown regulatory protein phosphorylated by CK2 $\alpha$; we postulate that PRR37 is a strong candidate for this unknown protein. Lin et al. (2000) reported that $\mathrm{Hd} 1$ genetically acts downstream of $\mathrm{Hd} 2$ to delay flowering time in LD, but no work has yet reported a direct link between these two CCTdomain containing proteins, $\mathrm{Hd} 1$ and PRR37, at the transcriptional or posttranslational level. For this reason, further work should examine the biochemical relationships of $\mathrm{Hd} 1$, PRR37, and $\mathrm{CK} 2 \alpha$ to reveal the molecular mechanism of $\mathrm{Hd} 6$-mediated floral repression in LD conditions. In addition, it would be worthwhile to examine whether $\mathrm{CK} 2 \alpha, \mathrm{CKI}$, or both can interact 


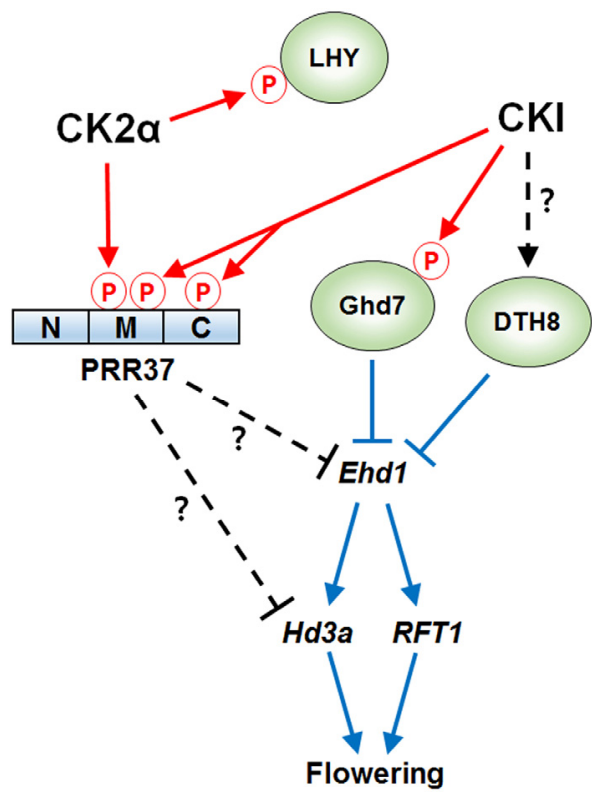

Fig. 5. Schematic model of regulation of PRR37 and other floral integrators by $\mathrm{CKI}$ and $\mathrm{CK} 2 \alpha$, in photoperiodic flowering under longday conditions in rice. Blue and red arrows indicate the regulation at the transcriptional and posttranslational levels, respectively.

with other OsPRR proteins such as OsTOC1/OsPRR1, OsPRR59, OsPRR73, and OsPRR95.

Second, Hd16 also acts as an LD-dependent floral repressor by downregulating Ehd1 expression (Hori et al., 2013; Kwon et al., 2014). Hori et al., (2013) used in vitro kinase assays to show that $\mathrm{CKI}$ interacts with and phosphorylates the LD-dependent floral repressor Ghd7, but does not phosphorylate Hd1. This suggests that $\mathrm{CKI}$ downregulates Ehd1 expression by upregulating Ghd7 activity at the posttranslational level. Similar to Hd6, Hd16 requires functional $H d 2$ to delay flowering in LD (Hori et al., 2013; Shibaya et al., 2011; Yammamoto et al., 2000), indicating that $\mathrm{Hd} 6, \mathrm{Hd} 16$, and $\mathrm{Hd} 2$ function in the same genetic pathway of LDdependent floral repression. Indeed, we found that CKI also interacts with and phosphorylates PRR37 (Figs. 1B, 2B, and 3B). These results strongly suggest that the posttranslational modification of PRR37 by CKI and CK2 $\alpha$ likely affects its activity and stability, which should be determined in vivo.

It is noteworthy that CKI and CK2 $\alpha$ phosphorylate distinct regions in the PRR37 protein; CKI phosphorylates the recombinant partial proteins PRR37m and PRR37c (Fig. 4B), but CK2 $\alpha$ phosphorylates only PRR37m (Fig. 4B). This suggests that the levels of phosphorylation by CKI and CK2 $\alpha$ might separately regulate the activity and/or stability of PRR37. Further in vitro kinase assays combined with site-specific mutagenesis of PRR37 will be necessary to identify the exact sites where CKI and CK2 $\alpha$ phosphorylate PRR37.

Natural variants of Hd1, PRR37, Ghd7, DTH8, Hd6, and Hd16 occur in the rice varieties that are currently cultivated in Asia and Europe, and these variants play important roles in the downregulation of Ehd1 expression to delay flowering in natural LD conditions (Fig. 5). Hd16/CKI inhibits flowering in the Ehd1related pathway through phosphorylation of Ghd7 (Hori et al., 2013) and PRR37. However, the relationship between PRR37 and Ehd1 remains unclear, based on two conflicting reports (Koo et al., 2013; Yan et al., 2013). Hd6/CK2 $\alpha$ might phosphor- ylate PRR37 to downregulate $\mathrm{Hd} 3 \mathrm{a}$ and $\mathrm{RFT} 1$ expression in the Hd1-related pathway (Ogiso et al., 2010) by as-yet unknown mechanisms. In addition, CK2 $\alpha$ phosphorylates OsLHY in vitro, although $\mathrm{Hd} 6 / \mathrm{CK} 2 \alpha$ is not involved in the circadian rhythm in rice (Ogiso et al., 2010). The LD-dependent repression of flowering by Ghd7 and PRR37 is genetically additive (Kim et al., 2013; Koo et al., 2013), indicating that both act independently and synergistically. DTH8 genetically acts downstream of Hd16 because dth8 is epistatic to Hd16 (Hori et al., 2013), and thus it remains to be elucidated whether CKI directly interacts with and phosphorylates DTH8, an OsHAP3 subunit of the CCAAT-box-binding transcription factor (Wei et al., 2010). Currently, japonica rice cultivars can grow in high-latitude regions up to $53^{\circ} \mathrm{N}$ because they can flower extremely early under natural LD conditions (>14 $\mathrm{h}$ light/day) during the short summer period (Izawa 2007). Natural variations in the two major LD-dependent floral repressors PRR37 and Ghd7 are associated with seasonal and regional adaptation of rice to growth in the northernmost areas (Koo et al., 2013; Xue et al., 2008). In the northernmost rice cultivation regions, nonfunctional alleles of prr37 and ghd7 are broadly distributed in rice cultivars, including 'Iburiwase', 'Hoshinoyume', and 'H143' (Fujino and Sekiguchi, 2005; Koo et al., 2013; Shibaya et al., 2011) (Supplementary Table S2). Notably, northern-limit rice cultivars have different combinations of $H d 6$ and $H d 16$ alleles. For example, Iburiwase has functional alleles of $\mathrm{Hd} 6$ and Hd16, Hoshinoyume has nonfunctional $h d 6$ and functional $\mathrm{Hd} 16$ alleles, and $\mathrm{H} 143$ has functional Hd6 and nonfunctional hd16 alleles (Fujino and Sekiguchi, 2005; Kwon et al., 2014; Nonoue et al., 2008; Shibaya et al., 2011) (Supplementary Table S2). However, these japonica rice cultivars exhibit the same early flowering time [about 75 days to heading (DTH)] under natural LD conditions in Suwon, Korea $\left(37^{\circ} \mathrm{N}\right)$, strongly supporting the hypothesis that the effects of Hd6 and Hd16 on floral repression depend completely on functional PRR37 and Ghd7. Interestingly, an elite japonica rice cultivar 'Koshihikari' (about 105 DTH) has functional PRR37 and Ghd7, and nonfunctional hd6 and hd16 alleles, and thus flowers earlier than 'Milyang23' (about 117 DTH), which has functional PRR37, Ghd7, Hd6, and Hd16 alleles (Koo et al., 2013; Kwon et al., 2014; Matsubara et al., 2008a) (Supplementary Table S2). In the absence of CKI and CK2 $\alpha$ functions, however, Koshihikari flowered much later than lburiwase, Hoshinoyume, and H143 (105 vs. 75 DTH), but similar to the prr37-KO mutant in the 'Dongjin' background under LD (92 vs. 93 DTH) and SD (71 vs. 70 DTH) conditions (Koo et al., 2013). Although Koshihikari and Dongjin have different genetic backgrounds, it is notable that both japonica cultivars have been bred to grow in temperate regions and they have similar photoperiod sensitivities ( 21 vs. 23 days). Thus, it can be speculated that, at least in part, PRR37 may not be functional in Koshihikari, similar to the prr37-KO mutant.

In conclusion, we propose that $\mathrm{CKI}$ and $\mathrm{CK} 2 \alpha$ may contribute to enhancing the photoperiod sensitivity of rice through phosphorylation of PRR37. Furthermore, our results provide new, important insights into CKI and/or CK2-mediated phosphorylation of PRR proteins in other plants including barley, wheat, sorghum, maize, and Arabidopsis.

Note: Supplementary information is available on the Molecules and Cells website (www.molcells.org).

\section{ACKNOWLEDGMENTS}

We thank Dr. Sangkee Rhee at Seoul National University for donating the modified pET28a plasmid and Mr. Yong-Jae Kim 
at the National Center for Inter-University Research Facilities (NCIRF) for technical assistance in CLSM analysis. This work was supported by the National Research Foundation of Korea (NRF) grant funded by the Korean government (MEST) (No. 2011-0017308).

\section{REFERENCES}

Abe, M., Kobayashi, Y., Yamamoto, S., Daimon, Y., Yamaguchi, A., Ikeda, Y., Ichinoki, H., Notaguchi, M., Goto, K., and Araki, T. (2005). FD, a bZIP protein mediating signals from the floral pathway integrator FT at the shoot apex. Science 309, 10521056.

Alabadi, D., Oyama, T., Yanovsky, M.J., Harmon, F.G., Mas, P., and Kay, S.A. (2001). Reciprocal regulation between TOC1 and LHY/CCA1 within the Arabidopsis circadian clock. Science 293, 880-883.

Beales, J., Turner, A., Griffiths, S., Snape, J.W., and Laurie, D.A. (2007). A pseudo-response regulator is misexpressed in the photoperiod insensitive Ppd-D1a mutant of wheat (Triticum aestivum L.). Theor. Appl. Genet. 115, 721-733.

Citovsky, V., Lee, L.Y., Vyas, S., Glick, E., Chen, M.H., Vainstein, A., Gafni, Y., Gelvin, S.B., and Tzfira, T. (2006). Subcellular localization of interacting proteins by bimolecular fluorescence complementation in planta. J. Mol. Biol. 362, 1120-1131.

Corbesier, L., Vincent, C., Jang, S.H., Fornara, F., Fan, Q.Z., Searle, I., Giakountis, A., Farrona, S., Gissot, L., Turnbull, C., et al. (2007). FT protein movement contributes to long-distance signaling in floral induction of Arabidopsis. Science 316, 1030-1033.

Dai, C., and Xue, H.W. (2010). Rice early flowering1, a CKI, phosphorylates DELLA protein SLR1 to negatively regulate gibberellin signalling. EMBO J. 29, 1916-1927.

Daniel, X., Sugano, S., and Tobin, E.M. (2004). CK2 phosphorylation of CCA1 is necessary for its circadian oscillator function in Arabidopsis. Proc. Natl. Acad. Sci. USA 101, 3292-3297.

Doi, K., Izawa, T., Fuse, T., Yamanouchi, U., Kubo, T., Shimatani, Z., Yano, M., and Yoshimura, A. (2004). Ehd1, a B-type response regulator in rice, confers short-day promotion of flowering and controls FT-like gene expression independently of Hd1. Gene Dev. 18, 926-936.

Farre, E.M., and Kay, S.A. (2007). PRR7 protein levels are regulated by light and the circadian clock in Arabidopsis. Plant $\mathrm{J}$. $52,548-560$

Fujino, K., and Sekiguchi, H. (2005). Mapping of QTLs conferring extremely early heading in rice (Oryza sativa L.). Theor. Appl. Genet. 111, 393-398.

Fujino, K., Yamanouchi, U., and Yano, M. (2013). Roles of the Hd5 gene controlling heading date for adaptation to the northern limits of rice cultivation. Theor. Appl. Genet. 126, 611-618.

Fujiwara, S., Wang, L., Han, L.Q., Suh, S.S., Salome, P.A. McClung, C.R., and Somers, D.E. (2008). Post-translational regulation of the Arabidopsis circadian clock through selective proteolysis and phosphorylation of pseudo-response regulator proteins. J. Biol. Chem. 283, 23073-23083.

Gao, H., Zheng, X.M., Fei, G.L., Chen, J., Jin, M.N., Ren, Y.L., Wu W.X., Zhou, K.N., Sheng, P.K., Zhou, F., et al. (2013). Ehd4 Encodes a Novel and Oryza-Genus-Specific Regulator of Photoperiodic Flowering in Rice. PLoS Genet. 9, e1003281

Hayama, R., Yokoi, S., Tamaki, S., Yano, M., and Shimamoto, K. (2003). Adaptation of photoperiodic control pathways produces short-day flowering in rice. Nature 422, 719-722.

Herrero, E., Kolmos, E., Bujdoso, N., Yuan, Y., Wang, M.M., Berns, M.C., Uhlworm, H., Coupland, G., Saini, R., Jaskolski, M., et al (2012). EARLY FLOWERING4 recruitment of EARLY FLOWERING3 in the nucleus sustains the Arabidopsis circadian clock. Plant Cell 24, 428-443.

Hori, K., Ogiso-Tanaka, E., Matsubara, K., Yamanouchi, U., Ebana, K., and Yano, M. (2013). Hd16, a gene for casein kinase I, is involved in the control of rice flowering time by modulating the day-length response. Plant J. 76, 36-46.

Huq, E., Tepperman, J.M., and Quail, P.H. (2000). GIGANTEA is a nuclear protein involved in phytochrome signaling in Arabidopsis. Proc. Natl. Acad. Sci. USA 97, 9789-9794.

Ito, S., Kawamura, H., Niwa, Y., Nakamichi, N., Yamashino, T., and Mizuno, T. (2009). A genetic study of the Arabidopsis circadian clock with reference to the TIMING OF CAB EXPRESSION 1 (TOC1) Gene. Plant Cell Physiol. 50, 290-303.

Izawa, T. (2007). Adaptation of flowering-time by natural and artificial selection in Arabidopsis and rice. J. Exp. Bot. 58, 3091-3097.

Kaczorowski, K.A., and Quail, P.H. (2003). Arabidopsis PSEUDORESPONSE REGULATOR7 is a signaling intermediate in phytochrome-regulated seedling deetiolation and phasing of the circadian clock. Plant Cell 15, 2654-2665.

Kang, C.H., Moon, B.C., Park, H.C., Koo, S.C., Chi, Y.H., Cheong, Y.H., Yoon, B.D., Lee, S.Y., and Kim, C.Y. (2013). Rice small C2-domain proteins are phosphorylated by calcium-dependent protein kinase. Mol. Cells 35, 381-387.

Kardailsky, I., Shukla, V.K., Ahn, J.H., Dagenais, N., Christensen, S.K., Nguyen, J.T., Chory, J., Harrison, M.J., and Weigel, D. (1999). Activation tagging of the floral inducer FT. Science 286, 1962-1965.

Kim, S.L., Choi, M., Jung, K.H., and An, G. (2013). Analysis of the early-flowering mechanisms and generation of T-DNA tagging lines in Kitaake, a model rice cultivar. J. Exp. Bot. 64, 4169-4182.

Knippschild, U., Gocht, A., Wolff, S., Huber, N., Lohler, J., and Stoter, M. (2005). The casein kinase 1 family: participation in multiple cellular processes in eukaryotes. Cell. Signal. 17, 675-689.

Kobayashi, Y., Kaya, H., Goto, K., Iwabuchi, M., and Araki, T. (1999). A pair of related genes with antagonistic roles in mediating flowering signals. Science 286, 1960-1962.

Komiya, R., Ikegami, A., Tamaki, S., Yokoi, S., and Shimamoto, K. (2008). Hd3a and RFT1 are essential for flowering in rice. Development 135, 767-774.

Komiya, R., Yokoi, S., and Shimamoto, K. (2009). A gene network for long-day flowering activates RFT1 encoding a mobile flowering signal in rice. Development 136, 3443-3450.

Koo, B.H., Yoo, S.C., Park, J.W., Kwon, C.T., Lee, B.D., An, G. Zhang, Z.Y., Li, J.J., Li, Z.C., and Paek, N.C. (2013). Natura variation in OsPRR37 regulates heading date and contributes to rice cultivation at a wide range of latitudes. Mol. Plant 6, 18771888.

Kwon, C.T., Yoo, S.C., Koo, B.H., Cho, S.H., Park, J.W., Zhang Z.Y., Li, J.J., Li, Z.C., and Paek, N.C. (2014). Natural variation in early flowering1 contributes to early flowering in japonica rice under long days. Plant Cell Environ. 37, 101-112.

Lin, H.X., Yamamoto, T., Sasaki, T., and Yano, M. (2000). Characterization and detection of epistatic interactions of 3 QTLs, Hd1, Hd2, and Hd3,controlling heading date in rice using nearly isogenic lines. Theor. Appl. Genet. 101, 1021-1028.

Lu, S.X., Liu, H., Knowles, S.M., Li, J., Ma, L., Tobin, E.M., and Lin, C. (2011). A role for protein kinase casein kinase2 alphasubunits in the Arabidopsis circadian clock. Plant Physiol. 157, 1537-1545.

Matsubara, K., Kono, I., Hori, K., Nonoue, Y., Ono, N., Shomura, A., Mizubayashi, T., Yamamoto, S., Yamanouchi, U., Shirasawa, K., et al. (2008a). Novel QTLs for photoperiodic flowering revealed by using reciprocal backcross inbred lines from crosses between japonica rice cultivars. Theor. Appl. Genet. 117, 935-945.

Matsubara, K., Yamanouchi, U., Wang, Z.X., Minobe, Y., Izawa, T., and Yano, M. (2008b). Ehd2, a rice ortholog of the maize INDETERMINATE1 gene, promotes flowering by up-regulating Ehd1. Plant Physiol. 148, 1425-1435.

Matsubara, K., Yamanouchi, U., Nonoue, Y., Sugimoto, K., Wang, Z.X., Minobe, Y., and Yano, M. (2011). Ehd3, encoding a plant homeodomain finger-containing protein, is a critical promoter of rice flowering. Plant J. 66, 603-612.

Matsubara, K., Ogiso-Tanaka, E., Hori, K., Ebana, K., Ando, T., and Yano, M. (2012). Natural variation in Hd17, a homolog of Arabidopsis ELF3 that is involved in rice photoperiodic flowering Plant Cell Physiol. 53, 709-716.

Matsushika, A., Makino, S., Kojima, M., and Mizuno, T. (2000) Circadian waves of expression of the APRR1/TOC1 family of pseudo-response regulators in Arabidopsis thaliana: Insight into the plant circadian clock. Plant Cell Physiol. 41, 1002-1012.

Mulekar, J.J., and Huq, E. (2014). Expanding roles of protein kinase CK2 in regulating plant growth and development. J. Exp. Bot. 65, 2883-2893

Murakami, M., Matsushika, A., Ashikari, M., Yamashino, T., and Mizuno, T. (2005). Circadian-associated rice pseudo response regulators (OsPRRs): Insight into the control of flowering time. Biosci. Biotech. Biochem. 69, 410-414. 
Murakami, M., Tago, Y., Yamashino, T., and Mizuno, T. (2007) Characterization of the rice circadian clock-associated pseudoresponse regulators in Arabidopsis thaliana. Biosci. Biotech. Biochem. 71, 1107-1110.

Murphy, R.L., Klein, R.R., Morishige, D.T., Brady, J.A., Rooney, W.L., Miller, F.R., Dugas, D.V., Klein, P.E., and Mullet, J.E. (2011). Coincident light and clock regulation of pseudoresponse regulator protein 37 (PRR37) controls photoperiodic flowering in sorghum. Proc. Natl. Acad. Sci. USA 108, 16469-16474.

Nakamichi, N., Kita, M., Ito, S., Yamashino, T., and Mizuno, T. (2005). PSEUDO-RESPONSE REGULATORS, PRR9, PRR7 and PRR5, together play essential roles close to the circadian clock of Arabidopsis thaliana. Plant Cell Physiol. 46, 686-698.

Nakamichi, N., Kita, M., Niinuma, K., Ito, S., Yamashino, T., Mizoguchi, T., and Mizuno, T. (2007). Arabidopsis clockassociated pseudo-response regulators PRR9, PRR7 and PRR5 coordinately and positively regulate flowering time through the canonical CONSTANS-dependent photoperiodic pathway. Plant Cell Physiol. 48, 822-832.

Nonoue, Y., Fujino, K., Hirayama, Y., Yamanouchi, U., Lin, S.Y. and Yano, M. (2008). Detection of quantitative trait loc controlling extremely early heading in rice. Theor. Appl. Genet. 116, 715-722.

Nusinow, D.A., Helfer, A., Hamilton, E.E., King, J.J., Imaizumi, T., Schultz, T.F., Farre, E.M., and Kay, S.A. (2011). The ELF4ELF3-LUX complex links the circadian clock to diurnal control of hypocotyl growth. Nature 475, 398-U161.

Ogiso, E., Takahashi, Y., Sasaki, T., Yano, M., and Izawa, T. (2010). The role of Casein Kinase II in Flowering Time Regulation Has Diversified during Evolution. Plant Physiol. 152, 808-820.

Park, D.H., Somers, D.E., Kim, Y.S., Choy, Y.H., Lim, H.K., Soh, M.S., Kim, H.J., Kay, S.A., and Nam, H.G. (1999). Control of circadian rhythms and photoperiodic flowering by the Arabidopsis GIGANTEA gene. Science 285, 1579-1582.

Park, S.J., Kim, S.L., Lee, S., Je, B.I., Piao, H.L., Park, S.H., Kim, C.M., Ryu, C.H., Park, S.H., Xuan, Y.H., et al. (2008). Rice indeterminate 1 (Osld1) is necessary for the expression of Ehd1 (Early heading date 1) regardless of photoperiod. Plant J. 56, 1018-1029.

Portoles, S., and Mas, P. (2010). The functional interplay between protein kinase CK2 and CCA1 transcriptional activity is essential for clock temperature compensation in Arabidopsis. PLoS Genet. 6, e1001201

Saito, H., Ogiso-Tanaka, E., Okumoto, Y., Yoshitake, Y., Izumi, H., Yokoo, T., Matsubara, K., Hori, K., Yano, M., Inoue, H., et al (2012). Ef7 encodes an ELF3-like protein and promotes rice flowering by negatively regulating the floral repressor gene Ghd7 under both short- and long-day conditions. Plant Cell Physiol. 53, 717-728.

Salome, P.A., and McClung, C.R. (2005). PSEUDO-RESPONSE REGULATOR 7 and 9 are partially redundant genes essential for the temperature responsiveness of the Arabidopsis circadian clock. Plant Cell 17, 791-803.

Sawa, M., Nusinow, D.A., Kay, S.A., and Imaizumi, T. (2007). FKF1 and GIGANTEA complex formation is required for day-length measurement in Arabidopsis. Science 318, 261-265.

Shibaya, T., Nonoue, Y., Ono, N., Yamanouchi, U., Hori, K., and Yano, M. (2011). Genetic interactions involved in the inhibition of heading by heading date QTL, Hd2 in rice under long-day conditions. Theor. Appl. Genet. 123, 1133-1143.

Song, Y.H., Smith, R.W., To, B.J., Millar, A.J., and Imaizumi, T. (2012). FKF1 conveys timing information for CONSTANS stabilization in photoperiodic flowering. Science 336, 1045-1049.

Sugano, S., Andronis, C., Green, R.M., Wang, Z.Y., and Tobin, E.M (1998). Protein kinase CK2 interacts with and phosphorylates the Arabidopsis circadian clock-associated 1 protein. Proc. Natl. Acad. Sci. USA 95, 11020-11025.

Takahashi, Y., Shomura, A., Sasaki, T., and Yano, M. (2001). Hd6, a rice quantitative trait locus involved in photoperiod sensitivity encodes the alpha subunit of protein kinase CK2. Proc. Natl. Acad. Sci. USA 98, 7922-7927.

Tan, S.T., Dai, C., Liu, H.T., and Xue, H.W. (2013). Arabidopsis casein kinase1 proteins CK1.3 and CK1.4 phosphorylate cryptochrome2 to regulate blue light signaling. Plant Cell 25, 2618-2632.
Taoka, K., Ohki, I., Tsuji, H., Furuita, K., Hayashi, K., Yanase, T., Yamaguchi, M., Nakashima, C., Purwestri, Y.A., Tamaki, S., et al. (2011). 14-3-3 proteins act as intracellular receptors for rice Hd3a florigen. Nature 476, 332-U397.

Tsuji, H., Tamaki, S., Komiya, R., and Shimamoto, K. (2008) Florigen and the photoperiodic control of flowering in rice. Rice 1, 25-35.

Turner, A., Beales, J., Faure, S., Dunford, R.P., and Laurie, D.A. (2005). The pseudo-response regulator $\mathrm{Ppd}-\mathrm{H} 1$ provides adaptation to photoperiod in barley. Science 310, 1031-1034.

Wang, L., Fujiwara, S., and Somers, D.E. (2010). PRR5 regulates phosphorylation, nuclear import and subnuclear localization of TOC1 in the Arabidopsis circadian clock. EMBO J. 29, 1903-1915.

Wei, X.J., Jiang, L., Xu, J.F., Zhang, W.W., Lu, G.W., Zhang, Y.S. and Wan, J.M. (2008). Genetic analyses of heading date of Japonica rice cultivars from Northeast China. Field. Crop. Res. 107, 147-154

Wei, X.J., Xu, J.F., Guo, H.N., Jiang, L., Chen, S.H., Yu, C.Y., Zhou, Z.L., Hu, P.S., Zhai, H.Q., and Wan, J.M. (2010). DTH8 suppresses flowering in rice, influencing plant height and yield potential simultaneously. Plant Physiol. 153, 1747-1758.

Wu, C.Y., You, C.J., Li, C.S., Long, T., Chen, G.X., Byrne, M.E., and Zhang, Q.F. (2008). RID1, encoding a Cys2/His2-type zinc finger transcription factor, acts as a master switch from vegetative to floral development in rice. Proc. Natl. Acad. Sci. USA 105, 12915-12920.

Wu, W.X., Zheng, X.M., Lu, G.W., Zhong, Z.Z., Gao, H., Chen, L.P., Wu, C.Y., Wang, H.J., Wang, Q., Zhou, K.N., et al. (2013) Association of functional nucleotide polymorphisms at DTH2 with the northward expansion of rice cultivation in Asia. Proc. Natl. Acad. Sci. USA 110, 2775-2780.

Xue, W.Y., Xing, Y.Z., Weng, X.Y., Zhao, Y., Tang, W.J., Wang, L., Zhou, H.J., Yu, S.B., Xu, C.G., Li, X.H., et al. (2008). Natura variation in Ghd7 is an important regulator of heading date and yield potential in rice. Nat. Genet. 40, 761-767.

Yamamoto, T., Lin, H., Sasaki, T., and Yano, M. (2000). Identification of heading date quantitative trait locus Hd6 and characterization of its epistatic interactions with $\mathrm{Hd} 2$ in rice using advanced backcross progeny. Genetics 154, 885-891.

Yamamoto, Y., Sato, E., Shimizu, T., Nakamich, N., Sato, S., Kato, T., Tabata, S., Nagatani, A., Yamashino, T., and Mizuno, T. (2003). Comparative genetic studies on the APRR5 and APRR7 genes belonging to the APRR1/TOC1 quintet implicated in circadian rhythm, control of flowering time, and early photomorphogenesis. Plant Cell Physiol. 44, 1119-1130.

Yan, W.H., Wang, P., Chen, H.X., Zhou, H.J., Li, Q.P., Wang, C.R Ding, Z.H., Zhang, Y.S., Yu, S.B., Xing, Y.Z., et al. (2011). A major QTL, Ghd8, plays pleiotropic roles in regulating grain productivity, plant height, and heading date in rice. Mol. Plant 4 , 319-330.

Yan, W.H., Liu, H.Y., Zhou, X.C., Li, Q.P., Zhang, J., Lu, L., Liu, T.M., Liu, H.J., Zhang, C.J., Zhang, Z.Y., et al. (2013). Natural variation in Ghd7.1 plays an important role in grain yield and adaptation in rice. Cell Res. 23, 969-971.

Yang, Y., Peng, Q., Chen, G.X., Li, X.H., and Wu, C.Y. (2013). OSELF3 is involved in circadian clock regulation for promoting flowering under long-day conditions in rice. Mol. Plant 6, 202-215.

Yano, M., Katayose, Y., Ashikari, M., Yamanouchi, U., Monna, L. Fuse, T., Baba, T., Yamamoto, K., Umehara, Y., Nagamura, Y., et al. (2000). Hd1, a major photoperiod sensitivity quantitative trait locus in rice, is closely related to the arabidopsis flowering time gene CONSTANS. Plant Cell 12, 2473-2483.

Youn, J.H., Kim, T.W., Kim, E.J., Bu, S., Kim, S.K., Wang, Z.Y., and Kim, T.W. (2013). Structural and functional characterization of Arabidopsis GSK3-like kinase AtSK12. Mol. Cells 36, 564-570.

Yu, J.W., Rubio, V., Lee, N.Y., Bai, S.L., Lee, S.Y., Kim, S.S., Liu, L.J., Zhang, Y.Y., Irigoyen, M.L., Sullivan, J.A., et al. (2008). COP1 and ELF3 control circadian function and photoperiodic flowering by regulating Gl stability. Mol. Cell 32, 617-630.

Zhao, J.M., Huang, X., Ouyang, X.H., Chen, W.L., Du, A.P., Zhu, L., Wang, S.G., Deng, X.W., and Li, S.G. (2012). OsELF3-1, an ortholog of Arabidopsis EARLY FLOWERING 3, regulates rice circadian rhythm and photoperiodic flowering. PLoS One 7, e43705. 